\title{
X-Ray Structural Studies of Bilayers Composed of Monoalkyl- Monocation Type Artificial Amphiphiles
}

\author{
Akira Harada, Kenji OKUYAma, ${ }^{* *}$ Atsushi Kumano, \\ Tisato KAJIYAMA,* Motowo TAKAYANAGI, \\ and Toyoki KUNITAKE*** \\ Department of Applied Chemistry and *** Organic Synthesis, \\ Faculty of Engineering, Kyushu University, Hakozaki, \\ Higashi-ku, Fukuoka 812, Japan
}

(Received March 25, 1985)

\begin{abstract}
The membrane structure of hydrated monoalkyl-monocation type artificial amphiphiles was investigated by an X-ray diffraction method. The amphiphiles showed a thermotropic and lyotropic crystal-liquid crystal transition similar to that of phospholipids in the biological membranes. The artificial amphiphile/water system with various amphiphile concentrations in the region from $C=0.8$ to $C=0.1$ (weight fraction) gave basically the same X-ray diffraction patterns. A one-dimensional structural analysis of the membrane was carried out for the specimen of $C=0.5$. According to this analysis, the repeating period of $4.20 \mathrm{~nm}$ consists of the water layer $(0.52 \mathrm{~nm})$ and the bimolecular layer $(3.68 \mathrm{~nm})$ in which hydrocarbon chains from both surfaces of the bimolecular layer are mutually inserted and almost perpendicular to the surface. Considering the thickness of the water layer, water molecules seem to be packed neatly and fixed strongly by the hydrogen bonds with bromide anions which are located at the surfaces of the bimolecular layer. As the number of carbon atoms in the alkyl group changed to 10,12 , and 14 , the crystal-liquid crystal phase transition temperature was raised to 303,310 , and $332 \mathrm{~K}$, respectively. The thickness of the bimolecular layer increased linearly with $0.26 \mathrm{~nm}$ per two alkyl groups, while the thickness of the water layer remained constant $(c a .0 .52 \mathrm{~nm})$. The wide angle diffractions of 0.47 , 0.40 , and $0.38 \mathrm{~nm}$ were independent of the length of the alkyl chain. This indicates that the above three amphiphiles were very similar in molecular conformation and packing arrangement.
\end{abstract}

KEY WORDS Artificial Amphiphile / Bilayer / Liposome / X-Ray

Structural Analysis / Structure Amplitude / Hydrogen Bond / Long Period /

Phospholipids are one of the main constituents of biomembranes and have both thermotropic and lyotropic crystal-liquid crystal transitions in the lipid/water system. They construct a bimolecular layer which was confirmed by transmission electron micrographs ${ }^{1}$ and X-ray diffraction studies. ${ }^{2,3}$.

Kunitake et al. synthesized artificial amphiphiles composed of monoalkyl- or dialkyl hydrophobic groups, and various types of polar head groups as biological membrane models. ${ }^{4}$ The phase transition behavior and the permeation properties of water and ions have been studied extensively for some of the above artificial amphiphiles. ${ }^{5-7}$ The monoalkyl-monocation type artificial amphiphile has both thermotropic and lyotropic crystalliquid crystal phase transitions in the artificial amphiphile/water system that occur in a similar manner to those of a biomembrane. ${ }^{8}$ The long spacing, $L$ at $300 \mathrm{~K}$ increased monotonously with decrease of the amphiphile con-

* To whom correspondence should be addressed.

** Present address: Department of Polymer Engineering, Tokyo University of Agriculture and Technology, Koganei, Tokyo 184, Japan. 
centration until $C=0.95$, at which free water appears in the system, while $L$ kept constant $(4.20 \mathrm{~nm})$ in the lower concentration. It was confirmed from small angle X-ray scattering that the bimolecular lamellar structure was formed even in a very dilute aqueous solution $(C<0.1)$, compared with other surfaceactive agents which could form the bimolecular layer only at rather high concentration above $C=0.6 .^{9}$ Further, electron micrographs showed that the monoalkyl-monocation type artificial amphiphile formed the bimolecular lamellar structure even at $C=0.001 .^{10}$ This outstanding ability of the bilayer formation might be due to the existence of the rigid diphenylimino group in the mesogenic part. In this study, we investigated in greater detail the structure of the bimolecular membrane formed by monoalkyl-monocation type artificial amphiphiles.

\section{MATERIALS AND METHODS}

The monoalkyl-monocation type artificial amphiphiles of Figure 1 were synthesized by the method of Kunitake et $a l .^{4}$ The synthesized artificial amphiphiles were all $p$-trimethylammoniumbenzylidene- $p^{\prime}$-alkylaniline bromide $\left(\mathrm{C}_{n} \mathrm{BBN}^{+} 3 \mathrm{C}_{1} ; n=10,12,14\right)$ and each consisted of three parts: the hydrophilic ammonium head group, the mesogenic diphenylimino group and the hydrophobic monoalkyl group.

For the purpose of X-ray diffraction measurement, multilamellar dispersions of hydrated $\mathrm{C}_{n} \mathrm{BBN}^{+} 3 \mathrm{C}_{1}$ were prepared by evaporating water from a dilute aqueous solution of an artificial amphiphile after sonication treatment. X-ray diffraction patterns were photographed by a plate camera collimated with toroid mirror optics, ${ }^{11}$ using nickelfiltered $\mathrm{Cu}-K_{\alpha}$ radiation $(\lambda=0.1542 \mathrm{~nm})$ from X-ray generater (Rota Flex R-200). Helium gas was passed through the camera to avoid air scattering. Microdensitometry of the diffraction photographs was carried out using a

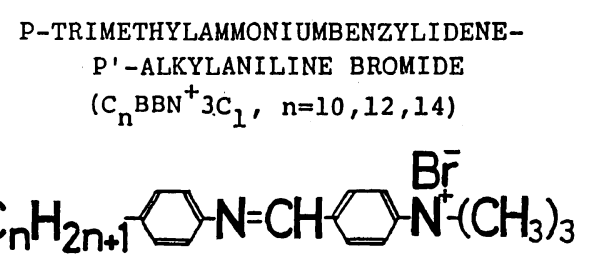

Figure 1. Chemical structure of artificial lipids.

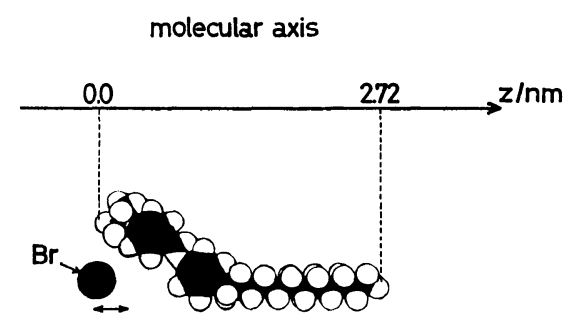

Figure 2. Molecular model of $\mathrm{C}_{\mathrm{i}_{2}} \mathrm{BBN}^{+} 3 \mathrm{C}_{1}$.

Joyce-Loebl film scanner. Baseline profiles were determined from traces on either side of each diffraction. The area $\left(A_{n}\right)$, in which the subscript " $n$ " indicated the $n$-th order diffraction, was converted to the relative structure amplitude, $F_{n}$, using the following relationship,

$$
F_{n}=n \sqrt{A_{n}}
$$

which provided approximate Lorentz and polarization corrections. ${ }^{12}$

Figure 2 shows the molecular model of $\mathrm{C}_{12} \mathrm{BBN}^{+} 3 \mathrm{C}_{1}$. The alkyl group was assumed to be all trans-conformation. The $z$-axis was set along the direction of the alkyl chain and its origin was located on one of the hydrogen atoms in the ammonium head group. Plausible molecular conformations were generated systematically by changing dihedral angles in a mesogenic group. All atoms for each structural model were projected on the $z$-axis, and the coordinate of $j$-th atom $\left(z_{j}\right)$ was measured. The $z$-coordinate of the bromide anion (b) was changed from the origin to the location of the nitrogen atom in the polar head group.

To simplify the bilayer structure of hydrated $\mathrm{C}_{12} \mathrm{BBN}^{+} 3 \mathrm{C}_{1}$, we divided it into the two parts, a bimolecular layer and a water layer as shown 
in Figure 3. The $Z$-axis was taken perpendicular to the bilayer surface and its origin was located at the center of the water layer. The angle between the $z$-axis and the membrane surface defined by $\theta$ and the distance between two origins of $z$ - and $Z$-axis was designated as translation $(t)$, which corresponded to a half thickness of the water layer. Then, the coordinate $z_{j}$ was converted to $Z_{j}$ by the equation

$$
Z_{j}=z_{j} \sin \theta+t
$$

The structure amplitude of $n$-th order, $F_{n}$, was calculated using the relationships

$$
\begin{aligned}
& F_{n}=\sum_{j=1} f_{j} \cos \left(2 \pi s Z_{j}\right) \\
& s=2 \sin \left(\phi_{n}\right) / \lambda
\end{aligned}
$$

where $\phi_{n}$ is the Bragg angle for $n$-th order

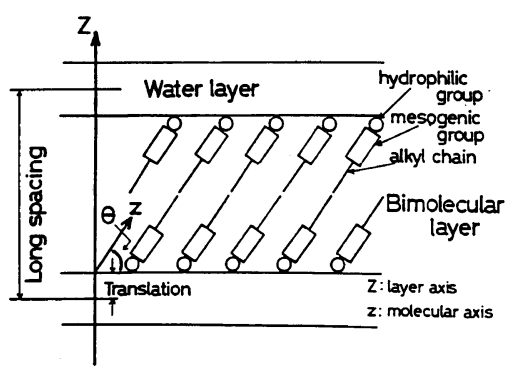

Figure 3. Schematic diagram of a lipid bilayer. diffraction, $\lambda$ is the wavelength of $\mathrm{Cu}-K_{\alpha}$ and $f_{j}$ is a water-weighted atomic scattering fac$\operatorname{tor}^{13,14}$ for the $j$-th atom. More than 12,000 structural models were generated by changing $\theta, t, b$, and the molecular conformation. Structure amplitudes, $F_{n}$, were calculated for each model and compared with the observed ones, $F_{n}^{0}$, in order to find the most plausible structure model.

\section{RESULTS AND DISCUSSION}

$\mathrm{X}$-Ray diffraction patterns were recorded from the $\mathrm{C}_{12} \mathrm{BBN}^{+} 3 \mathrm{C}_{1} /$ water system. Figure 4 shows the pattern for the hydrated $\mathrm{C}_{12} \mathrm{BBN}^{+} 3 \mathrm{C}_{1}$ at $C=0.51$ and $300 \mathrm{~K}$. Here, $C$ denotes the weight fraction of the artificial amphiphiles in aqueous solution. The diffraction patterns were almost identical at concentrations of $C=0.60,0.51,0.30$, and 0.15 . Six diffraction rings corresponding to a periodicity of $4.20 \mathrm{~nm}$ were observed, which meant that $\mathrm{C}_{12} \mathrm{BBN}^{+} 3 \mathrm{C}_{1}$ molecules aggregated into a lamellar form in the $\mathrm{C}_{12} \mathrm{BBN}^{+} 3 \mathrm{C}_{1} /$ water system. Diffraction with spacing shorter than $0.65 \mathrm{~nm}$ was not taken into account since it might be attributable to the lateral periodicity of the alkyl group or diphenylimino group. After Lorentz and po-

\section{$C=0.51(298 K)$}
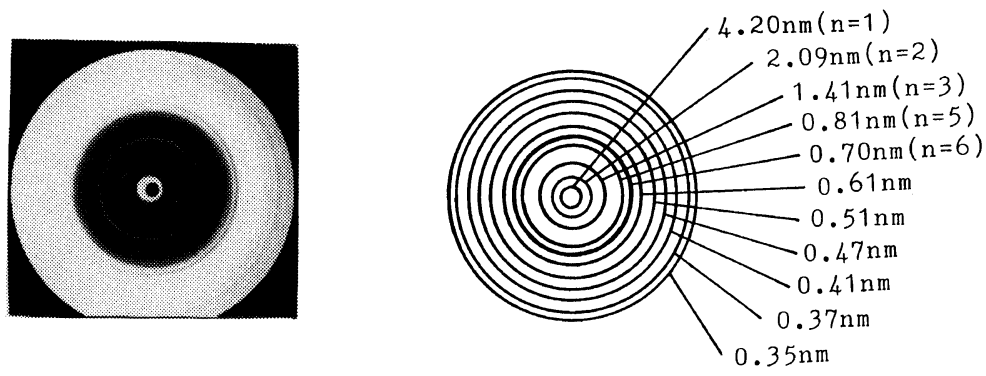

$F_{1}^{\circ}: F_{2}^{\circ}: F_{3}^{\circ}: F_{4}^{\circ}: F_{5}^{\circ}: F_{6}^{\circ}=100: 10: 21: 0: 42: 50$

Figure 4. $\mathrm{X}$-Ray diffraction pattern of $\mathrm{C}_{12} \mathrm{BBN}^{+} 3 \mathrm{C}_{1}(C=0.51)$ and its schematic diagram. 
A. Harada et al.

Table I. Observed $\left(E_{n}^{0}\right)$ and calculated $\left(F_{n}\right)$ structure amplitudes for $\mathrm{C}_{12} \mathrm{BBN}^{+} 3 \mathrm{C}_{1}{ }^{\mathrm{a}}$

\begin{tabular}{|c|c|c|c|c|c|c|c|c|c|}
\hline$\frac{t}{\mathrm{~nm}}$ & $\frac{b}{\mathrm{~nm}}$ & $\frac{\theta}{\circ}$ & $F_{1}$ & $F_{2}$ & $F_{3}$ & $F_{4}$ & $F_{5}$ & $F_{6}$ & $R^{\mathrm{b}}$ \\
\hline \multirow{5}{*}{0.26} & \multirow{5}{*}{$0 . \theta 0$} & 70 & 100 & 23 & 16 & 5 & 49 & 65 & 0.18 \\
\hline & & 75 & 100 & 24 & 19 & 3 & 51 & 63 & 0.18 \\
\hline & & 80 & 100 & 25 & 20 & 1 & 53 & 61 & 0.17 \\
\hline & & 85 & 100 & 25 & 22 & 0 & 54 & 60 & 0.17 \\
\hline & & 90 & 100 & 25 & 22 & 0 & 54 & 59 & 0.17 \\
\hline \multirow{3}{*}{0.16} & \multirow{3}{*}{0.10} & 80 & 100 & 27 & 14 & 5 & 46 & 80 & 0.28 \\
\hline & & 85 & 100 & 28 & 15 & 4 & 48 & 82 & 0.30 \\
\hline & & 90 & 100 & 28 & 15 & 4 & 49 & 82 & 0.30 \\
\hline
\end{tabular}

a $F_{1}^{0}: F_{2}^{0}: F_{3}^{0}: F_{4}^{0}: F_{5}^{0}: F_{6}^{0}=100: 10: 21: 0: 42: 50$.

b $R=\sum_{n}|| F_{n}^{0}|-| F_{n}|| / \sum_{n}\left|F_{n}^{0}\right|$.

larization corrections, the relative structure amplitudes, $F_{1}, F_{2}, F_{3}, F_{4}, F_{5}$, and $F_{6}$ were $100,10,21,0,42$, and 50 , respectively.

The models with good agreement between the observed structure amplitude, $F_{n}^{0}$ and that calculated, $F$, were examined by changing the parameters of $\theta, t, b$, and dihedral angles, systematically. Changes in the dihedral angles in the mesogenic group gave only a little effect on their $z$-coordinates. Therefore, the variation of the molecular conformation was not so sensitive to the magnitudes of structure amplitudes. Table I shows several examples with fairly good agreement between observed and calculated structure amplitudes. Among them, the model with $t=0.26 \mathrm{~nm}, b=0.00 \mathrm{~nm}$, and $\theta=85^{\circ}$ or $90^{\circ}$ gave the best agreement. This model is shown in Figure 5, in which the repeating period of $4.20 \mathrm{~nm}$ consists of the bimolecular layer $(3.68 \mathrm{~nm})$ and water layer $(0.52 \mathrm{~nm})$. The hydrocarbon chains attached to the polar heads on different surfaces were mutually inserted from opposite directions and were almost perpendicular to the membrane surface $\left(\theta \simeq 90^{\circ}\right)$ as shown in Figure 5 . Bromide anions were located at the surface of the bimolecular layer. Considering the thickness of the water layer ( $c a .0 .5 \mathrm{~nm}$ ) and also, the hydrogen bond length between water and $\mathrm{Br}^{-}(c a .0 .33 \mathrm{~nm}),{ }^{15}$ the water molecules seem

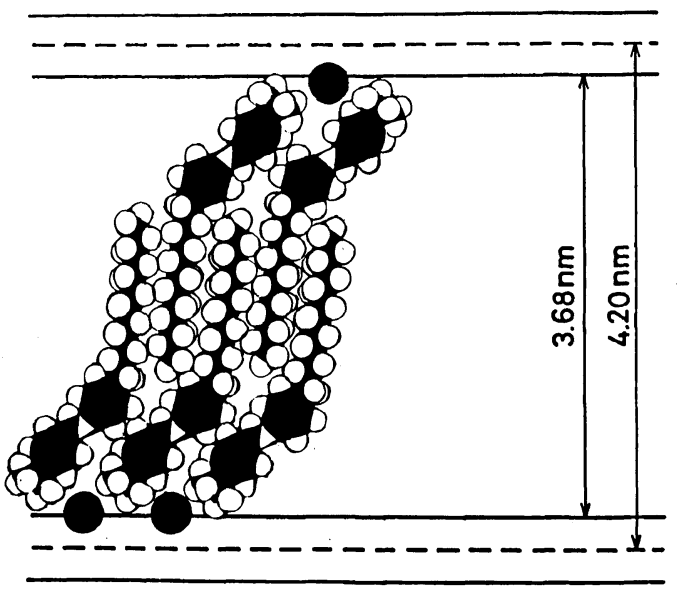

Figure 5. Representative structure of the most plausible model.

to be packed neatly and fixed strongly by the hydrogen bonds as schematically illustrated in Figure 6.

$\mathrm{C}_{10} \mathrm{BBN}^{+} 3 \mathrm{C}_{1}$ and $\mathrm{C}_{14} \mathrm{BBN}^{+} 3 \mathrm{C}_{1}$ showed similar $\mathrm{X}$-ray diffraction patterns to that of $\mathrm{C}_{12} \mathrm{BBN}^{+} 3 \mathrm{C}_{1}$. Also, they exhibited similar thermotropic and lyotropic liquid crystal phase transition behavior to that of $\mathrm{C}_{12} \mathrm{BBN}^{+} 3 \mathrm{C}_{1}$. Table II shows the crystal-liquid crystal phase transition temperatures, $T_{\mathrm{c}}$ and the long spacings for both powders $(C=1.0)$ and the fully hydrated state $(C=0.1)$ of $\mathrm{C}_{n} \mathrm{BBN}^{+} 3 \mathrm{C}_{1} . T_{\mathrm{c}}$ of the hydrated $\mathrm{C}_{n} \mathrm{BBN}{ }^{+} 3 \mathrm{C}_{1}$ were 303,310 , and 


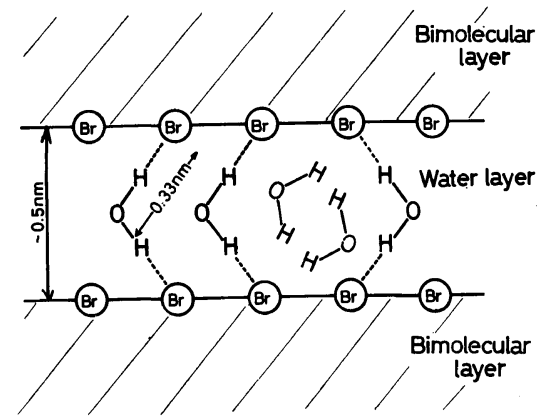

Figure 6. Hydrogen bond between $\mathrm{Br}^{-}$ion and water in the lipid bilayer.

Table II. The crystal-liquid crystal phase transition temperature of the fully hydrated state and long spacings for both powders $(C=1.0)$ and one of the fully hydrated $C_{n} \mathrm{BBN}^{+} 3 \mathrm{C}_{1}$

\begin{tabular}{|c|c|c|c|c|}
\hline \multirow{2}{*}{ Lipids } & \multirow{2}{*}{$\frac{T_{\mathrm{C}}^{*}}{\mathrm{~K}}$} & \multirow{2}{*}{$\frac{\begin{array}{c}\text { Bimolecular } \\
\text { length }\end{array}}{\mathrm{nm}}$} & $\begin{array}{l}\text { Long s } \\
C=0.1\end{array}$ & $\begin{array}{l}\text { pacing } \\
C=1.0\end{array}$ \\
\hline & & & $\mathrm{nm}$ & $\mathrm{nm}$ \\
\hline $\mathrm{C}_{10} \mathrm{BBN}^{+} 3 \mathrm{C}_{1}$ & 303 & 5.1 & 3.94 & 2.70 \\
\hline $\mathrm{C}_{12} \mathrm{BBN}^{+} 3 \mathrm{C}_{1}$ & 310 & 5.6 & 4.20 & 2.72 \\
\hline $\mathrm{C}_{14} \mathrm{BBN}^{+} 3 \mathrm{C}_{1}$ & 332 & 6.1 & 4.46 & 2.94 \\
\hline
\end{tabular}

$332 \mathrm{~K}$ for $n=10, n=12$, and $n=14$, respectively. Since an increase of the number of carbon atoms in alkyl chain group leads to an increases of lateral hydrophobic interaction among alkyl chains, it is reasonable that $T_{\mathrm{c}}$ increase with the number of carbon atoms.

Figure 7 shows the long spacings, $L$ for $\mathrm{C}_{n} \mathrm{BBN}^{+} 3 \mathrm{C}_{1}(n=10,12$, and 14$)$ together with their $\mathrm{X}$-ray diffraction patterns. As in the case of $\mathrm{C}_{12} \mathrm{BBN}+3 \mathrm{C}_{1}$, the X-ray patterns from the fully hydrated $\mathrm{C}_{n} \mathrm{BBN}^{+} 3 \mathrm{C}_{1}(n=10,14)$ were independent of the free water concentration. The long spacings obtained from the hydrated $\mathrm{C}_{n} \mathrm{BBN}^{+} 3 \mathrm{C}_{1}$ with $n=10$ and $n=14$ were $3.94 \mathrm{~nm}$ and $4.46 \mathrm{~nm}$, respectively. As seen in Figure 7, the long spacing monotonously increases with the number of $\mathrm{CH}_{2}$ groups in the hydrocarbon chain. The magnitude of increasing in a long period was $0.52 \mathrm{~nm}$ for four methylene groups. If methylene chains are in a planar zigzag conformation and also aligned

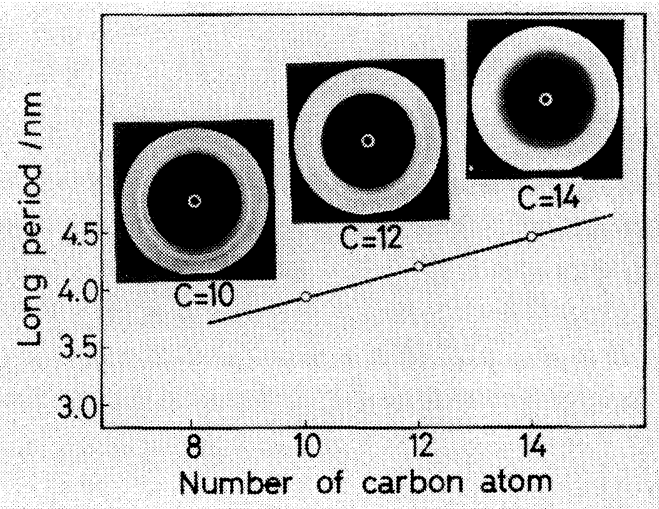

Figure 7. Long periods and X-ray diffraction patterns of $\mathrm{C}_{n} \mathrm{BBN}^{+} 3 \mathrm{C}_{1}(n=10,12$, and 14$)$.

perpendicular to the membrane surface, the difference of a long period between $\mathrm{C}_{n} \mathrm{BBN}^{+} 3 \mathrm{C}_{1}$ with $n=10$ and $n=14$ corresponds to the magnitude mentioned above. This indicates that the aggregation state of methylene chains shown in Figure 5 is fairly certain. Further, the patterns from these analogues had the same diffractions at $0.47,0.40$, and $0.38 \mathrm{~nm}$ which were expected from the lateral ordering of the alkyl chains. Therefore, these analogues are assumed to have very similar molecular conformations and packing states within the bimolecular layer. The observed structure amplitudes of $\mathrm{C}_{n} \mathrm{BBN}^{+} 3 \mathrm{C}_{1}$ were

$$
\begin{aligned}
& F_{1}^{0}: F_{2}^{0}: F_{3}^{0}: F_{4}^{0}: F_{5}^{0}: F_{6}^{0} \\
& \quad=100: 12: 27: 0: 68: 59 \quad \text { for } n=10
\end{aligned}
$$

and also,

$$
\begin{aligned}
& F_{1}^{0}: F_{2}^{0}: F_{3}^{0}: F_{4}^{0}: F_{5}^{0}: F_{6}^{0} \\
& \quad=100: 18: 25: 10: 38: 58 \text { for } n=14
\end{aligned}
$$

The structural analyses of $\mathrm{C}_{n} \mathrm{BBN}^{+} 3 \mathrm{C}_{1}$ $(n=10,14)$ were carried out by the same method as in the case of $\mathrm{C}_{12} \mathrm{BBN}^{+} 3 \mathrm{C}_{1}$. The most plausible structures of fully hydrated $\mathrm{C}_{n} \mathrm{BBN}^{+} 3 \mathrm{C}_{1}(n=10,14)$ were fundamentally similar to that of $\mathrm{C}_{12} \mathrm{BBN}^{+} 3 \mathrm{C}_{1}$ except for the thickness of the bimolecular layer. On the other hand, thickness of the water layer was 


\section{A. Harada et al.}

kept constant (ca. $0.5 \mathrm{~nm})$. Similar linear relationships between the thickness of the bimolecular layer and number of carbon atoms have been observed for 1,2-diacyl-L-phosphatidylcholine, 1,2-diacyl-DL-phosphatidylethanolamine, 1,2-diacyl-L-phosphtidyl-L-serines, 1,2- and 1,3-diacyl-DL-glycero-phosphatidic acid, fatty acids, diglycerides, and triglycerides, for which the number of alkyl group ranges from $10<n<20{ }^{16}$

In conclusion, the $\mathrm{X}$-ray diffraction patterns of hydrated monoalkyl-monocation type artificial amphiphiles were independent of their concentrations in the region from $C=0.1$ to $C=0.8$. The structural analyses for $\mathrm{C}_{n} \mathrm{BBN}^{+} 3 \mathrm{C}_{1}$ in the fully hydrated state indicated that the hydrophobic alkyl chains were aligned almost perpendicular to the membrane surface $\left(\theta \simeq 90^{\circ}\right)$ and stacked in the fashion of the mutual insertion of hydrocarbon chains extending from opposite directions. The thickness of the bimolecular layer increased with the length of the alkyl chain, while the thickness of the water layer kept constant at about $0.5 \mathrm{~nm}$. The lateral ordering or packing of alkyl chains in the bimolecular layer was independent of the length of the alkyl chain.

\section{REFERENCES}

1. L. M. Rzepecki, J. Berriman, and J. B. Finean,
Biochim. Biophys. Acta, 600, 72 (1980).

2. R. H. Pearson and I. Pascher, Nature, 281, 449 (1979).

3. I. Sakurai, S. Iwayanagi, T. Sakurai, and T. Seto, $J$. Mol. Biol., 117, 285 (1977).

4. T. Kunitake, Y. Okahata, K. Tamaki, F. Kumamaru, and M. Takayanagi, Chem. Lett., 387 (1977).

5. T. Kajiyama, A. Kumano, M. Takayanagi, Y. Okahata, and T. Kunitake, Chem. Lett., 645 (1979).

6. T. Kunitake and Y. Okahata, J. Am. Chem. Soc., 102, 549 (1980).

7. A. Kumano, T. Kajiyama, M. Takayanagi, T. Kunitake, and Y. Okahata, Ber. Bunsenges. Phys. Chem., 88, 1216 (1984).

8. A. Kumano, T. Kajiyama, M. Takayanagi, T. Kunitake, and Y. Okahata, Bull. Chem. Soc. Jpn., 58, 1205 (1985).

9. V. Luzzati and F. Husson, J. Cellular Biol., 12, 207 (1962).

10. T. Kajiyama, A. Kumano, M. Takayanagi, Y. Okahata, and T. Kunitake, Contemporary Topics in Polym. Sci., 4, 829 (1984).

11. A. Elliot, J. Sci. Instrum., 42, 312 (1965).

12. M. H. F. Willkins, A. E. Blaurock, and D. M. Engeiman, Nature New Biology, 230, 72 (1971).

13. K. Okuyama, N. Tanaka, T. Ashida, and M. Kakudo, Bull. Chem. Soc. Jpn., 49, 1805 (1976).

14. R. D. B. Fraser, T. P. Macrae, and E. Suzuki, J. Appl. Cryst., 11, 693 (1978).

15. G. C. Pimentel and A. L. McClellan, "The Hydrogen Bond," W. H. Freeman and Company, San Francisco, 1960.

16. G. C. Kresheck, "Water: A Comprehensive Treatise," Vol. 4, F. Franks, Ed, Plenum Press, New York, 1975, Chapter 2. . 